\title{
Learning from robotic artefacts: A quest for strong concepts in Human-Robot Interaction
}

\author{
Nazli Cila \\ Department of Human-Centered \\ Design, Delft University of \\ Technology \\ The Netherlands \\ n.cila@tudelft.nl
}

\author{
Cristina Zaga \\ Human-Centred Design Group, \\ University of Twente \\ The Netherlands \\ c.zaga@utwente.nl
}

\author{
Maria Luce Lupetti \\ Department of Human-Centered \\ Design, Delft University of \\ Technology \\ The Netherlands \\ m.l.lupetti@tudelft.nl
}

\begin{abstract}
This paper is a methodological replication of Barendregt et al. [11], who urged Child-Computer Interaction field to embrace Intermediate Level Knowledge as a meaningful and valid way of generating knowledge. We extend this epistemological gap to the Human-Robot Interaction (HRI). Currently, artefact-centered papers-papers that present the development of an artefact-seem to be one of the primary ways that the HRI field generates knowledge. In this paper, we made an analysis of all papers presented at the HRI Conference from 2006 to 2020. Our results indicate that the $41,2 \%$ of the papers were artefact-centered; and the impact of them, measured in the number of citations, was significantly lower than other kinds of papers. We used 23 artefact-centered papers to formulate two strong concepts and investigate how the foundational design epistemology about intermediate-level knowledge and RtD can contribute to other design-related disciplines to produce useful and valuable knowledge.
\end{abstract}

\section{CCS CONCEPTS}

- Human-centered computing $\rightarrow$ Interaction design theory, concepts and paradigms.

\section{KEYWORDS}

Intermediate-level knowledge, Strong concepts, Human-Robot Interaction, Artefact-centered paper, Epistemology

\section{ACM Reference Format:}

Nazli Cila, Cristina Zaga, and Maria Luce Lupetti. 2021. Learning from robotic artefacts: A quest for strong concepts in Human-Robot Interaction. In Designing Interactive Systems Conference 2021 (DIS '21), fune 28-fuly 2, 2021, Virtual Event, USA. ACM, New York, NY, USA, 10 pages. https: //doi.org/10.1145/3461778.3462095

\section{INTRODUCTION}

In 2010, Eric Stolterman and Mikael Wiberg observed that it was a very common trend at Human-Computer Interaction (HCI) conferences that the researchers presented artefacts that manifested some new or improved form of interactivity [93]. These designs were

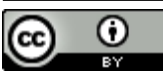

This work is licensed under a Creative Commons Attribution International 4.0 License.

DIS '21, June 28-fuly 2, 2021, Virtual Event, USA

(C) 2021 Copyright held by the owner/author(s)

ACM ISBN 978-1-4503-8476-6/21/06.

https://doi.org/10.1145/3461778.3462095 usually based on earlier work from the field; some evaluations were conducted; and the results were presented with some suggestions for future work. Stolterman and Wiberg [93] argued that "these presentations do not leave a lasting impression on the audience of fellow researchers, as the contributions do not address, challenge, or complement the existing body of theoretical knowledge within interaction research in an intentional way" (p. 96). With a similar observation, in 2017, Wolmet Barendregt and her colleagues made a "call for action" to the Child-Computer Interaction (CCI) community to embrace intermediate-level knowledge in their ways of working [11]. They argued that artefact-centered papers-the papers that describe the design process and evaluation of a particular artefact-present only "design instances", which are unique and stand-alone products in the vast space of other possible solutions. In line with [93], they maintained that these papers make a smaller contribution to the field as it is hard for other researchers to build further upon the kind of knowledge presented. Intermediate-level knowledge (i.e., representations of knowledge in-between general theories and design instances [54]) and strong concepts (i.e., a form of intermediate-level knowledge which consists of design elements that are abstracted from an artefact and can be applied to other applications and use situations [54]) are proposed as a way to support the CCI field evolve as a discipline.

As design researchers who mostly work at the intersection of design and human-robot interaction (HRI), we recognize the same epistemological problem identified by [11] and [93]. Looking at the flagship ACM/IEEE International Conference on Human-Robot Interaction, for instance, we notice that many presentations comply with the conventional way that the HRI community produces knowledge: defining a problem or function, building an artefact or interaction, testing, analyzing, and repeating from the beginning until satisfied [13]. These artefacts are usually technologically advanced and valuable solutions for specific problems; however, they carry the risk that only a small part of the knowledge gained from exploring this problem can be carried over to the next one.

The trail that had started from HCI and led to CCI takes us to HRI in this paper. The paper is a "methodological replication" of the study of Barendregt et al. [11] for the context of HRI. We focus on the ACM/IEEE International Conference on Human-Robot Interaction (henceforth HRI Conference) since it is the most mature HRI conference in terms of perceived quality [12], includes articles reflecting diverse approaches on HRI (e.g. empirical, conceptual, and methodological), and hosts a large number of articles with easily accessible citation and bibliometric data. We will replicate the 
research questions and methodology of [11] with slight adaptations. The key research questions are as follows:

- RQ 1. Which portion of the papers presented at the HRI Conference consists of artefact-centered papers?

- RQ 2. What impact do artefact-centered papers have in terms of citations when compared to other kinds of papers?

- RQ 3. Which defensible, contestable, and substantive strong concepts can be generated by analyzing the currently available artefact-centered papers in HRI?

Concepts underlying this paper build on prior works describing how new knowledge can be reflectively produced from design work, and more generally, ongoing inquiries in the HCI field into the role of design as key means in knowledge construction activities, i.e., research-through-design (RtD) [14, 44, 62, 92, 104]. RtD is focused on "making the right thing", as opposed to "making the thing right"; yet in the field of HRI, much of the research efforts go into the latter [72]. It is our intention to show that RtD could enable building a knowledge base that other researchers can refer to and build upon in HRI, which is one of the current shortcomings in the field [31].

Human-robot interaction is considered to be fundamentally different from human-computer interaction as HRI deals with complex, dynamic control systems that exhibit autonomy and cognition, and operate in changing, real-world environments [88]. We acknowledge this difference for sure, yet our aim in this paper is to draw (the missing) parallels between the epistemologies of these two fields. The HRI community could enormously benefit from adopting new ways of generating knowledge that go beyond "the only one 'recipe' for how to get accepted by the HRI conference" ([32], p. 4:1) in order to further the knowledge-oriented discourse in the community. The approach proposed in this article is about providing conceptual tools (in the form of intermediate-level knowledge) to the HRI researchers to explore what HRI design epistemology is and could be. We also intend this paper to contribute to the DIS community by expanding the ongoing inquiries on RtD to a related ACM field and issue an invitation for the ways in which HCI researchers may be productively intertwined in this knowledge-generation process.

In the remainder of this paper, we will first shortly discuss the fundamentals of knowledge production in design and how it is different from the HRI tradition. We will then describe what constitutes intermediate-level knowledge, and how strong concepts can be generated and assessed. Thereafter, we will describe the review process of the HRI conference papers, and present two strong concepts-grounding of communication and same vs. other-extracted from the artefact-centered papers. The paper concludes with discussing the generative potential of strong concepts and how to build bridges between the HCI and HRI disciplines.

\section{DESIGN THEORY AND INTERMEDIATE-LEVEL KNOWLEDGE}

The claim that design is a discipline in its own right [27] signified a breaking point in the history of design research. There was a movement away from early technically-oriented approaches for improving the methods and processes of design, towards a comprehension of design as a cognitive and social, creative reflective practice [28] (see [25] for a history of design research). The discipline's knowledge bases, underlying skill-sets and values were identified, articulated, and clarified [69]. Design claimed its own epistemics in the construction of knowledge. Despite these academic achievements and the establishment of a genuine discipline of design, the field has been struggling with the alleged disciplinary distribution of tasks where makers make and theorists theorize, as if the two were completely separate matters of concern [80]. The twin pillars of design research-"design" and "research"-seemed to be in conflict with one another. The particularity of design [78] was considered to be incompatible with the generalizing tendencies of research [10, 30, 54, 93]. Lloyd summarized this tension as:
"There is a sense in which design research sits at the crux of a false dichotomy; between on the one hand research in a "pure" form (which values objectivity, experiment, discourse, history, analysis) and on the other the active engagement in shaping future forms by suggestion, prototype, speculation, practice, and intervention at all levels (...)" [68]

To overcome this dichotomy, some scholars chose to assert that the norms and values underlying scientific problem solving and design problem solving were the same, uniting them through a shared core cognitive process (e.g. [40]). Others were engaged in creating alternative theories of inquiry to bring design and research into alignment. In this new framing, the accumulation of design research deeply committed to practices of making and the increasing presence of more conceptual and discursive approaches to design [80]. Under the notion of RtD, designing and making had become central to how the research process unfolds, and thus to what constitutes the production of new knowledge. Indeed, Basballe and Halskov [14] show that design and research in RtD continuously couple, interweave, and decouple. Knowledge is inherent to the activity of designing itself (gained through engaging in and reflecting on the activity) or to the design artefacts (gained through reflecting on those artefacts). For this reason, the artefacts produced in this type of research can open up unanticipated design spaces [46], be vehicles for theory building [80,101], and/or invite debate around issues [38]. In other words, they become "epistemic objects" [39], "concept-things" [79], or "research artefacts" [104], which become a conduit for transferring the research findings to research and design communities.

In HRI, on the other hand, the role of artefacts has been mainly about solving concrete problems (e.g. how to make the mobile robot mission specification system more user-friendly, how to improve team performance in a joint human-robot task, how a robot should approach two people, and so on). The main methodology of the field is empirical. For example, Bartneck [12] showed that the empirical work outnumbered any other type of work at the HRI conference between 2009-2014. Similarly, Baxter et al. [15] found out that 96 out of 101 papers from the same conference between 2013-2015 were experimental papers. The primary hypotheses that are tested in HRI are related to providing an exploration or account of some human-centered phenomenon, or improving the functionalities and application of the robot (ibid). Obviously, this empirical approach does not negate RtD (see the "Lab" approach in [62]), yet the multiple roles that robotic artefacts can play in the generation of knowledge and the other explorative RtD methods are still relatively unknown to the majority of HRI design researchers, as 
emphasized also in the limited number of contributions found on this topic $[66,70,72,76]$. Still, the field recognizes the need to embrace new methods, methodologies, and in general a theoretical and conceptual basis, in order to establish itself as a research field alongside HCI or psychology [31]. This is where intermediate-level knowledge comes into play.

\subsection{Intermediate-level knowledge}

In their seminal paper from 2012, Höök and Löwgren [54] argued that HCI research mainly produced knowledge on the levels of instances and theories, using a predominantly empirical approach. Instances has to do with the highly situated, specific, and contextual knowledge related to a particular artefact or situation. Theories are about abstracted knowledge that is universally true and applicable. On the one hand, instances are rarely considered sufficient in themselves to form new knowledge; on the other, the abstraction from situatedness and specificity seems to lose the compelling qualities that designs can offer [29]. To bridge abstract knowledge and situated outcomes without disregarding the qualities of design practice, Höök and Löwgren [54] suggested intermediate-level knowledge forms, which explain more than a set of designs but not aiming at producing general theories. Intermediate-level knowledge is more abstracted than particular instances, yet does not aspire to the generality of a theory (ibid).

Stolterman and Wiberg similarly proposed the concept of "constructs" (i.e., an idea or theory containing various conceptual elements, typically one considered to be subjective and not based on empirical evidence) as a midway between a single concept and an all-encompassing theory [93]. As they state, "Instead of being intimidated by the expectations of building theory, interaction researchers can, through the concept-driven approach, focus on combining individual theoretical concepts into constructs that bring together earlier findings in new concepts and artifacts" (p. 112). Both constructs and intermediate-level knowledge are a rich means to allow discussions on paradigmatic systems and exemplary artefacts that go beyond specific functions and look-and-feel of the system [93], advance the discourse on interaction design epistemology, and strengthen the rigor in design-oriented research.

Examples of intermediate-level knowledge include patterns, guidelines, annotated portfolios, methods and tools, experiential qualities, design heuristics, criticism, strong concepts, manifestos, design programs, and bridging concepts [54]. The adoption of these knowledge forms in HRI is at a nascent state. Elsewhere, we made an extensive overview on how they are currently employed in HRI [71], yet a few examples include "design patterns" created for describing the interaction of a robot with a child within a particular context [59], "heuristics" such as continuous actions and boundary signaling to improve the robots' social acceptability [34], and "criticism" on representing the divine in robots [98].

In this paper, we specifically focus on strong concepts. Since it is our aim in this paper to investigate the potential of generating knowledge from already existing artefact-centered papers (i.e., instance-level), strong concepts are good candidates for this purpose with the reasons to become clear in the next section.

\subsection{Strong Concepts}

Strong concepts are design elements or principles that are generative, namely, they can be used by other designers/researchers to create instances in different design situations [54]. Component(s) of a particular artefact can be isolated and abstracted to the level that it can be realized in many different ways in a whole class of applications, a range of use situations, or different genre of designs (ibid). Artefact-centered papers could thus potentially contain the basis for one or more strong concepts.

Höök and Löwgren [54] describe how such knowledge construction can be undertaken as follows: The first step is to identify the elements in the instance that could be valuable for other design situations within the same domain or that cross the domain's boundaries. Then, the elements are linked to similar concepts, i.e., horizontal grounding, in order to scope the range of applicability of the strong concept. Then vertical grounding is conducted by examining whether the strong concept is present in other known instances and the theories that the strong concept is a concretization of. The final step involves validating whether the strong concept is contestable, defensible, and substantive. Being contestable refers to suggesting a way of seeing things that has not already been well-established in the existing literature of the academic community in question [50]. Being defensible refers to being grounded empirically, analytically, and theoretically; and that the community could accept the new knowledge given the arguments or evidence. Lastly, being substantive refers to the contribution being relevant for the community in question. For strong concepts in particular, substantivity has to do with its potential to be used in designing new instances [54].

In the next section, we will present how we identified the artefactcentered papers in the HRI Conference and explain how we followed the process described by Höök and Löwgren to extract contestable, defensible, and substantive strong concepts.

\section{METHOD}

In order to seek answers to the research questions, a sample consisting of research articles from the HRI Conference from 2006 to 2020 was derived. We exclusively focused on research articles as they are thoroughly peer-reviewed, and excluded demonstrations, late-breaking works, workshop proposals, student design competitions, and video presentations. This gave a sample of 587 full papers, distributed unequally over time. To develop the coding scheme, we adapted the description of Barendregt et al. [11] on artefact-centered papers as follows:

(1) The main object of discussion of the paper is a concrete functional artefact developed within a research project. We defined the artefacts in the HRI context to be robots/robotic objects, robot morphologies, behaviors, interaction capabilities, interfaces, datasets, software, algorithms, and systems. In other words, concrete entities that are part of a robot's form and functioning were included.

(2) The creators of the artefacts were involved in writing the paper, and therefore, the evaluation includes original work and not commercial robots.

(3) The paper typically presents a background (e.g. theories, previous work by other inspiring the design of the artefact), 
design process (e.g. user studies, co-design activities, prototypes), the actual artefact, the artefact's evaluation (e.g. computer simulations, user tests, field studies), and optionally some generalizations in the form of recommendations or guidelines of directions for future work.

The papers that do not belong to this category are human-robot interaction user studies (i.e., how humans perceive, interact or live with robots) and theory and methods in HRI (i.e., new theoretical concepts, fundamental HRI principles beyond individual interfaces, methods used for investigating various aspects of human-robot interactions).

All articles were coded using the definition above to determine which papers were artefact-centered (RQ 1). In addition, meta-data on citation number, publication year, and authorship were recorded. The coding was done via reading the abstracts, and when necessary the full texts. Each article took an average of 5 mins. Fatigue effects were mitigated by limiting coding to a maximum of 60 articles per day. The coding schema was discussed among all authors regarding its application in HRI and to clarify the concepts. The first author coded all papers from 2020 (around 10\% of the whole sample, as recommended by [51]) and prepared a summative table to display which papers from the corpus were considered to be artefact-centered. Then, the three authors had an open discussion about the results of this pilot coding. The main discrepancy had to with the different ways that the authors defined what an artefact is in this context. One of the authors focused on the visual/embodied components of a robot (e.g. form, interface, movement); whereas the other two also considered its technical components (e.g. algorithms, models, software) as an artefact. We decided to keep the latter broad description of an artefact. Every instance of disagreement was resolved in the order they appeared in the summative table. Based on this negotiated agreement procedure, we considered the coding reliability to be suitable for further analysis. All coding was carried out by the first author.

To answer RQ 2, we used the number of citations for each paper as indicated in the ACM Digital Library in December 2020. While citation, as a proxy for research impact, does have some limitations; it is widely accepted [74], also in design research [16, 20, 21]. Due to the unequal sizes of the two groups ( $\mathrm{N}$ of artefact-centered papers $=242$, $\mathrm{N}$ of others $=345$ ), we used Welch's unequal variances t-test to compare the number of citations.

Regarding RQ 3, again following the methodology of [11], we identified the artefact-centered papers with unusually high citations to extract strong concepts. Potentially, all artefact-centered papers can contain strong concepts. However, the papers that were cited more than average stand as stronger candidates since other scholars were able to benefit from those studies (as indicated by the citation count). Since RQ 3 is an investigation into determining whether it is possible to generate contestable, defensible, and substantive strong concepts from existing artefact-centered papers, we considered these papers to be a sufficient sample. In order to identify the artefact-centered papers with unusually high number of citations, we first determined the mean and the standard deviation of the number of citations for each year. Using these, we selected all artefact-centered papers that were cited more than 1 standard deviation above the mean number of citations for the year that paper was published. Papers from 2019 and 2020 were excluded from this analysis as only very few citations were yet available.

The selection procedure resulted in 23 papers. Following the strong concept generation process of Höök and Löwgren [54] described in the previous section, each of these papers were read in detail by one of the authors, who noted down some potential elements in the artefacts that could be of value in other design situations. Later, the authors discussed these strong concepts they identified, as well as the possible overlaps among the papers which would highlight the importance of those particular strong concepts even more [11]. Based on this discussion, two strong concepts that covered several of the papers were chosen.

\section{RESULTS}

\subsection{Percentage of artefact-centered papers in the HRI Conference}

RQ 1 involved investigating which portion of the HRI Conference papers were artefact-centered. The distribution of the percentages for each year can be seen in Figure 1 (range $=30 \%-54 \%$ ). The combined mean of all years is $41.2 \%$, which indicates that a significant portion of all papers at the HRI Conference was focused on a particular artefact. This number is in line with the $42 \%$ that Barendregt et al. [11] had found in relation to the number of artefact-centered papers in the CCI Conference between 2003-2016 .

\subsection{Impact of artefact-centered papers}

RQ 2 sought to find out the relative impact of the artefact-centered papers in the ACM community. A Welch two-samples t-test revealed that the difference between the artefact-centered papers and the other kinds of papers was statistically significant $(t=2.49, p<$ $0.01)$, meaning that the artefact-centered papers have been cited less $(M=18.7, S D=23.6)$ than the other kinds $(M=24.9, S D=35.9)$. This result indicates that artefact-centered papers have indeed a lower impact in the field, in line with the findings of Barendregt et al. [11]. It also corresponds with the studies in design theory, which show that increasing levels of theory development predicts significant increases in citation count (e.g. [16]).

\subsection{Strong concepts in the artefact-centered papers}

RQ 3 involved investigating which defensible, contestable, and substantive strong concepts could be generated from already existing artefact-centered papers at the HRI conference. 23 artefact-centered papers with unusually high number of citations (i.e., more than 1 standard deviation above the mean for the artefact-centered papers of that year) were chosen (Table 1). Theoretically, every artefactcentered paper could include one or more strong concepts. Yet, the strength of strong concepts comes from revealing the common ideas and patterns that exist within a knowledge field. Although we were able to identify multiple strong concepts from the 23 artefact-centered papers in our corpus (e.g. legibility of motion, predictability, socially appropriate behavior), we decided to pursue two primary strong concepts as they showed the highest overlap between the papers, namely "grounding of communication" and "same vs. other". The papers which these strong concepts were 


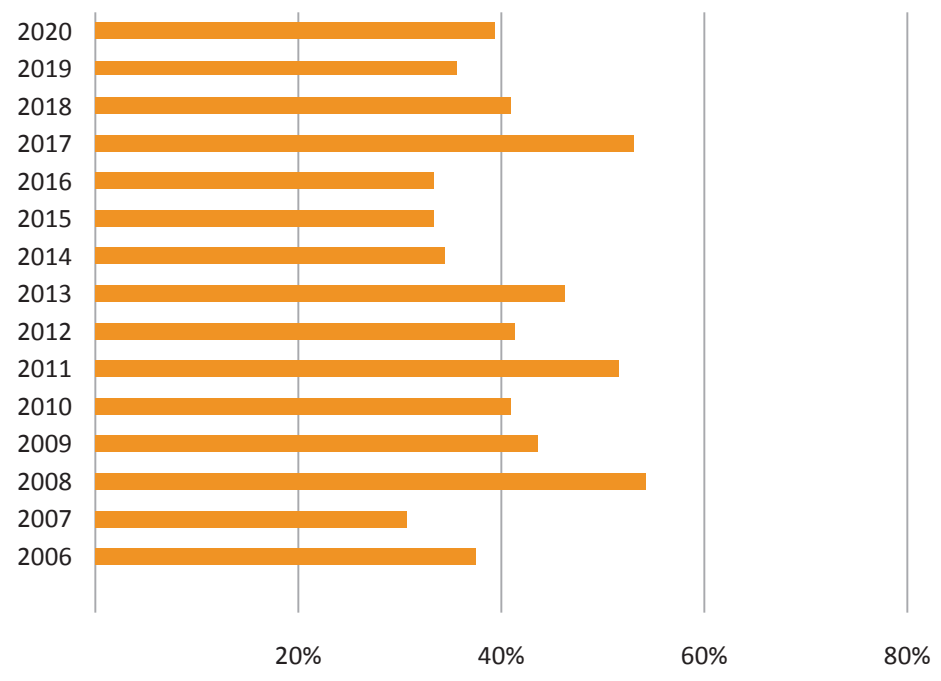

Figure 1: Percentage of artefact-centered papers in the HRI Conference between 2006-2020

extracted from are indicated in Table 1 with an asterisk. In order to show that these strong concepts have the potential to be contestable, defensible, and substantive, we will make some first steps towards vertical and horizontal grounding in the next section.

4.3.1 Grounding Communication. Mutating the definition from [24] and [97], by grounding of communication, we refer to the ability to nonverbally coordinate the content and process of communication, particularly mutual knowledge of each other's presence, coordination of each other's attention, redirection of each other's attention to a reference object, or place of interest in the context of communication and coordination of state, intention, and emotional expression. It is a fundamental collaborative feature of human communication that humans practice explicitly and implicitly when interacting with other humans and animals [97].

Humans ground their communication in many ways: by adding deixis (i.e., words to refer to time, place, and context like "here", "there", "yesterday", or nonverbal behaviors like pointing), backchanneling (i.e., verbal interjection like "uh-oh", or nonverbal interjection like nodding), focusing, directing and redirecting attention using gaze movements or gestures (e.g. pointing, following each other's gaze), and making iconic or metaphoric gestures (i.e., pantomiming the physical representation of a concrete object or an abstract concept) [24, 47, 97]. In human-robot interaction and in particular social robotics, the grounding of communication is considered essential to organize the human-robot goal-directed behavior (e.g. going from A to B), and communicate intentions and affective states. Gaze, multimodal conversational gaze, gestures, conversational gestures, non-anthropometric intent communication are some of the behaviors that fall under grounding of communication in HRI.

Andrist et al. [6], in our corpus of artefact-centered papers, offer an example of grounding of communication strong concept related to human-like gaze aversion. The authors studied the design of gaze aversion, unveiling how it helps the robots to signal cognitive efforts and regulates the conversation between humans and robots both at the emotional (i.e., level of intimacy) and functional level (i.e., managing the conversation floor). Another example is by Huang and Mutlu [55], who demonstrated how dynamic Bayesian networks help coordinate multimodal behaviors (i.e., gestures, speech, and gaze) achieved natural content coordination during human-robot interaction. Szafir et al. [94] designed a natural and intuitive flight motion in order to communicate the intent of assistive free-flyers (AFFs). They show how specific motion parameters and manipulation of motion primitives of the AFFs help to coordinate the content and process of communication with co-located users.

Horizontal grounding: While the grounding of communication concept was established in the linguistics [24] and semiotics [47] for human-human interaction, the grounding of communication concept with agents and computers has been adopted in the HCI and Human-Agent Interaction (HAI) fields to understand and design computer- and agent-mediated communication. CSCW research has significantly focused on grounding, unveiling much of the mechanisms, issues, and computer-mediated grounding opportunities. For example, the seminal work of Cramton [26] demonstrated how additional "grounding" behaviors are needed when interacting via computer-mediated communication with dispersed collaborators, since talking through a computer makes mutual coordination of contextual knowledge difficult. Grounding is also fundamental in remote collaboration systems, such as remote gesturing [61], and in instant messaging systems to communicate affection [99]. In Human-Virtual Agents Interaction, grounding is used and studied with similar lenses as in human-like HRI research. For example, Andrist et al. [5] studied how hybrid stochastic models from humanhuman interaction can be applied successfully to virtual agents in order to coordinate communication.

Vertical grounding: In HRI, the properties, design principles, and implications of using gaze for grounding of human-robot communication have been studied from many angles (e.g. cognitive 
modelling, knowledge representation, behavior coordination, affective regulation, design of social cues, nonverbal communication). To give some examples, Lemaignan et al. [67] designed models for knowledge representation and spatial planning to ground a collaborative task. Ros et al. [82] investigated ways to disambiguate deictic communication to redirect attention to an object or subject. In this strong concept, gaze is arguably the cue and mechanism that it is studied with the most frequency. Admoni and Scasellati [2] compiled a review of how HRI employed social eye gaze of robots to coordinate social engagement, interaction and process. Mutlu [77] showed that gaze not only coordinated the grounding process, but actively grounded and shaped the content of human-robot conversations and tasks. The grounding property of gaze has been studied also for robots communicating in human-terms. Zaga et al. [103] showed that minimal gaze movements of a low-anthropomorphic robot are perceived by children as grounding social engagement, redirecting attention and being helpful. Building on Zaga's work, Anderson-Bashan et al. [4] showed that abstract gaze grounded people's perceptions of an abstract robot. Human-like robot gestures have been studied to replicate the grounding mechanism of human-like deictic and metaphoric gestures (e.g. [87]). Lastly, Jung [58] demonstrated how verbal and nonverbal behavior of a robot shaped the interactional management of affective grounding.

4.3.2 Same vs. Other. The other strong concept we identified from our corpus involves the ontology of robots, which we conceptualized as a continuum. On the one side, there is the concept of sameness, that is, the replication of human-human interaction modalities and dynamics as the determining factor for effective human-robot interactions. This concept relates to the broad theme of anthropomorphism and is grounded on the idea that if a robot acts like a human, human-robot interaction would be facilitated [13, 105]. The other end of the continuum, otherness, is about the peculiar non-human ways in which a robot can communicate. It is grounded on the idea that humans tend to interpret actions of things-even artificial-as intentional [49].

In our corpus, sameness can be seen in the work of Adalgeirsson and Breazeal [1], who demonstrated that a telepresence robot with human-like social expressions enabled more engaging and enjoyable interactions. Similarly, Shah and colleagues [89] showed that the performance of a human-robot team improved when the robot (i.e., Chaski) mimicked the behaviors and strategies observed in human teams. Huang and Mutlu [55] used a dynamic Bayesian network for modelling how humans coordinate speech, gaze, and gesture and integrated the model into a robot's behavior in order to enable natural, multimodal interactions. Falling in the scope of otherness, Saerbeck and Bartneck [85] revealed that some specific motion characteristics of a robot could work as indicators of physical and mental states. Song and Yamada [91] explored the use of unique colors, sounds, and vibrations to craft the expressiveness of simple-shaped robots. Similarly, Szafir et al. [95] developed a unique visual signaling mechanism for small flying-robots to communicate directionality and facilitate co-located interactions with people.

The concepts of sameness and otherness translate not only into different robot morphologies but also behaviors and roles; and these have advantages and limitations. For instance, sameness facilitates interactions that feel "natural" to humans; therefore, there is no additional cognitive load required from the users to interpret the actions of the robot. However, sameness can lead to uncanny feelings when it is at its extreme [75] or elicit unrealistic expectations from the robots, which often makes people to overestimate the robots' capabilities [102]. On the other hand, otherness supports calibrating the expectations of the users (see the case of the robotic vacuum cleaners eliciting low expectations in users [43]) and help constructing the "pretense" situation that the humans and robots are in [70]. However, at the same time it requires more interpretation and learning from the users, which might result in less effective interactions [4].

These two concepts, however, are not mutually exclusive. Much of the HRI work, in fact, presents aspects of sameness and otherness simultaneously. Satake et al. [86], for example, investigated the appropriate ways of approaching people in public spaces (e.g. shopping malls). The embodiment of the chosen robot (Robovie) had human-like features-the head and the arms performed human-like expressions; yet, the focus of the authors was on understanding the possible interaction patterns emerging from the specific humanrobot interplay.

Horizontal grounding: This strong concept is related to the scholarship in the disciplines of humanities, STS, and philosophy which has retired from perspectives that place human beings at its center. The approach of Actor-Network Theory [64, 65], Object-Oriented Ontology [18, 48], or Activity Theory [60] have been adopted by the HCI community to imagine alternative futures in which objects express agency in their own ways $[22,36,83]$ (see also "more than human design" [42, 45]). For example, Wakkary et al. [100] designed Morse Things-a set of bowls and cups that independently communicate with each other through Morse code-in order to challenge the human-centered focus in IoT and create a new understanding of the relationship between humans and technologies through a thingcentered approach. Similarly, Jenkins et al. [57] presented a set of digital artefacts in order to illustrate the ways in which computing's agency is made material. In Human-Virtual Agents Interaction, the tension between anthropomorphism and non-anthropomorphism has been also discussed in relation to avatars (e.g. [9]) and conversational agents (e.g. [7]).

Vertical grounding: Focusing on how the sameness and otherness concepts shape the embodiment of robots, one can find a wide spectrum of robotic artefacts in HRI: Robot replicas (e.g. the androids developed by Hiroshi Ishiguro [56]), anthropomorphic robots (e.g. Kismet [19] and Flobi [73]), hybrid robots (e.g. Keepon [63], Shybo [70], and MeBot [1]), minimalistic robots (e.g. The Greeting Machine [4], Kip1 [53], and Yolo [3]), and robjects (e.g. Mechanical Ottoman [90], MicBot [96], and Ranger [41]). These robots present a range from employing exact replicas of human-like features to minimalistic and abstracted human-like behaviors. Sameness and otherness also account for the interpretative processes that a human engages with when interacting with a robot. Seminal, in this regard, is the work of Hoffman and Ju [52] who argued that the robotic movements did not need to follow the human form in detail to be interpreted as intentional. Lastly, there are also some excellent speculative design projects that push the idea of "other" further. A classic example is the Technological Dreams Series by [37], which envision robots as technological cohabitants with their sometimes 


\begin{tabular}{|c|c|c|}
\hline Year & Title & Citations \\
\hline 2008 & A point-and-click interface for the real world: laser designation of objects for mobile manipulation & 68 \\
\hline 2008 & Understanding human intentions via hidden markov models in autonomous mobile robots & 70 \\
\hline 2009 & ${ }^{*}$ How to approach humans?: strategies for social robots to initiate interaction & 134 \\
\hline 2010 & An affective guide robot in a shopping mall & 131 \\
\hline 2010 & ${ }^{*}$ MeBot: a robotic platform for socially embodied presence & 46 \\
\hline 2010 & Recognizing engagement in human-robot interaction & 47 \\
\hline 2011 & ${ }^{*}$ Perception of affect elicited by robot motion & 44 \\
\hline 2011 & ${ }^{*}$ Improved human-robot team performance using chaski, a human-inspired plan execution system & 87 \\
\hline 2011 & Automatic analysis of affective postures and body motion to detect engagement with a game companion & 113 \\
\hline 2011 & A conversational robot in an elderly care center: an ethnographic study & 77 \\
\hline 2012 & Strategies for human-in-the-loop robotic grasping & 82 \\
\hline 2013 & Legibility and predictability of robot motion & 42 \\
\hline 2013 & Human-robot cross-training: computational formulation, modeling and evaluation of a human team training strategy & 22 \\
\hline 2014 & ${ }^{*}$ Conversational gaze aversion for humanlike robots & 86 \\
\hline 2014 & ${ }^{*}$ Learning-based modeling of multimodal behaviors for humanlike robots & 59 \\
\hline 2014 & ${ }^{*}$ Communication of intent in assistive free flyers & 72 \\
\hline 2015 & ${ }^{*}$ Communicating Directionality in Flying Robots & 69 \\
\hline 2015 & When Children Teach a Robot to Write: An Autonomous Teachable Humanoid Which Uses Simulated Handwriting & 70 \\
\hline 2016 & Design and Evaluation of a Rapid Programming System for Service Robots & 15 \\
\hline 2016 & Emotion Encoding in Human-Drone Interaction & 22 \\
\hline 2017 & ${ }^{*}$ Expressing Emotions through Color, Sound, and Vibration with an Appearance-Constrained Social Robot & 37 \\
\hline 2018 & Communicating Robot Motion Intent with Augmented Reality & 30 \\
\hline 2018 & Improving Collocated Robot Teleoperation with Augmented Reality & 30 \\
\hline
\end{tabular}

Table 2: Artefact-centered papers with an unusually high number of citations (according to the ACM Digital Library in December 2020)

"alien" means of communication. Amplifying the unique characteristics and "needs" of robots, Auger [8] focused on adapting the context to robots, instead of adapting the robots to the context.

\section{DISCUSSION AND CONCLUSION}

Throughout this article we have suggested intermediate-level knowledge forms as a necessary addition to broaden the scope of the HRI research. This work was a methodological replication of the [11] for the HRI domain, which revealed very similar results with the original article. We have shown that currently one of the primary means that the HRI field produces knowledge is by describing and evaluating a robot or a component of a robot, and that this kind of papers generally have a lower citation number (and indirectly, a lower impact) than their non-artefact-centered counterparts. We then attempted to create more abstracted, generalizable concepts from 23 of such papers. These strong concepts were "grounding of communication" and "same vs. other". Obviously, our aim was not to diminish the undeniable value of these studies and prescribe how researchers should have done their work. Rather, we intended to search for similar approaches researchers have taken in addressing common problems in order to generate a more abstracted and sharable HRI knowledge base. The artefacts in these artefact-centered papers reflected a specific framing of a particular problem, situated themselves in an ecology of other artefacts that adopt similar framings or use markedly different framings to address the same problem (e.g. anthropomorphism or non-anthropomorphism). We aimed to make these ecologies explicit in this work via the vertical and horizontal grounding for those strong concepts.

Before discussing the implications of this work, there are three relevant limitations to be considered. First, citations provide a widely recognized measure of scientific impact but are less suited to evaluating practical impact, for instance on patents, programming tools, or robot design practice. As our focus was on the scientific maturation of the HRI field, we did not focus on the knowledge uptake in practice. Yet, it may well be that (some of) the artefactcentered papers identified in this paper have been heavily supporting the HRI practice in companies/institutions. Therefore, this paper should be considered in conjunction with more practice-focused reflections. Second, corresponding with how theories are seen as something not always stable and constant but as something unfolding [80], strong concepts should also be described and discussed as provisional [54]. The two strong concepts we identified follow the spirit of the HRI field at this particular point in time, and may become irrelevant after a short time period. Still, this would not diminish the value of such an exercise to seek underlying, abstracted concepts between design instances. This brings us to the third limitation that the suggested strong concepts in this paper do not claim to cover all possible strong concepts that can be found through an analysis of the HRI material or that the vertical and horizontal grounding we have performed is complete [11]. Grounding requires a deep and broad knowledge of related concepts, instances, and theories, which would require a collective effort from the discipline 
(ibid). That is why the HRI field is in need of other intermediateknowledge forms such as annotated portfolios in order to identify common ideas and patterns in the field holistically [71].

Going back to the criteria for assessing strong concepts-being contestable, defensible, and substantive-we aimed to demonstrate in this paper how the strong concepts of grounding for communication and same vs. other could be of relevance and valuable for the field of HRI. Horizontal grounding serves the purpose of showing the roots of a particular concept and how it is addressed in other fields. For this reason, it helps to assess the novelty of the knowledge contribution (i.e., contestability) [54]. In our brief horizontal grounding attempts, we aimed to demonstrate this novelty by pointing out to the origin of these concepts in humanities and philosophy and making links to the HCI, HAI, and CSCW communities in order to describe how these concepts have been investigated in these sister-fields. Vertical grounding was intended for showing the defensibility, where the similar works of other scholars in HRI provide the "evidence" for the validity of these concepts. Lastly, maybe the most interesting one for the purposes of this paper, substantiveness is about the potential of the strong concept to be used in designing new instances [54]. We have provided examples of how the strong concepts have the potential to be generative at various levels. For example, grounding of communication can be generative both in the creation of design principles for grounding (e.g. gaze behaviors, affective grounding) and the generation of heuristics for grounding communication in HRI. In other words, when the researchers/designers are faced with a new design challenge concerning creating intimacy between a human and a robot, for instance, they can turn to the grounding of communication strong concept and use it as a starting point. Similarly, the concept of same vs. other could be purposefully addressed as a way of tackling the nuances in shifting perspectives from human to the non-human, and playing around with emulation or translation, and abstraction of human-human interaction modalities. The horizontal and vertical grounding behind these concepts, as well as the example applications of the concepts by others could also function as inspiration and reference [11]. One can use the two strong concepts identified above to build on the examples, employ the approaches in a future design problem, and eventually, leverage the knowledge created by the resulting artefacts.

However, strong concepts, and other forms of intermediate-level knowledge for that matter, are rarely addressed or still completely missing in the HRI community [71]. Our argument is that the conceptual value of these types of knowledge would make them particularly meaningful for advancing the disciplinary understanding of what a robot is, how it should interact with people, and what purposes it should serve. Gaver [44] gave various characteristics as to what design theory emerged through RtD could be. These are "generative", "suggestive", "provisional", "aspirational", "annotative", which point to a very different identity from the descriptive and empirical methodologies of the current HRI. Even though the field has started to welcome discussions on what HRI research is and the best ways of "doing" it, and the community members are urged to have an "open mind to adopt whatever methodology is appropriate for the particular research questions" [33, 44], accepting these new knowledge generation forms could be a challenge. As the DIS and CHI communities have already recognized RtD as a valid knowledge contribution, HRI researchers/designers can join forces with the $\mathrm{HCI}$ community to promote RtD and intermediatelevel knowledge in HRI. This entails discussing which RtD methods might be relevant for HRI and what sorts of contributions these could make in the scientific growth of the discipline. Furthermore, as Luria et al. point out, publishing about RtD on HRI topics in DIS and $\mathrm{CHI}$ conferences have the potential to create a growing group of researchers that are on the intersection of these fields and allow RtD to enter HRI more naturally [72], which was also one of the intentions behind this paper.

Another way forward for the HRI community is to demonstrate how original and transferable knowledge, such as strong concepts, has emerged through design processes. This requires a rigorous documentation of progress and evolution of artefacts. Such documentation would make it possible to see a chain of reasoning that leads to a final artefact $[17,23]$ and build clearer links between the artefact and the knowledge generated [35]. Furthermore, detailed descriptions of the design process and related micro-assumptions allow for replication (i.e., one of the main problems in the HRI field identified by [31]) and bring transparency and rigor to the whole research process. The lack of proper documentation is a problem even in the HCI community [10, 17, 23, 92], with only few models meant for scholarly reporting of the RtD activities, e.g. [10, 79, 84]. Desjardins and Key [35] discuss that the RtD process is more like "a mesh of lines that cross" than a "chain". The artefacts of RtD emerge within and throughout the mesh, instead of appearing as an end point. HRI, on the other hand, currently have a linear way of generating knowledge (or at least this is how it is presented in the scholarly publications), probably because of the discipline's roots in engineering and psychology. The organic configurations that $\mathrm{RtD}$ enable for producing knowledge may become a challenge for the field to accept. Yet, we strongly advocate that engaging with questions such as how the work was done and which kind of underlying assumptions and questions were explored would enable the HRI researchers to foster awareness regarding the rationale behind their specific design choices and identify the actions that correspond with these.

This paper is an invitation to the HRI community to engage in conversations with the DIS and CHI communities, get inspired by the evolutionary broadening that the these communities went through in the past decade [81] to establish RtD as a valid means for generating knowledge [92], and collaborate within or outside of the discipline boundaries to find out how bridging towards design research would produce intermediate-level knowledge [54]. All these efforts would support the HRI field to move forward, create a foundation of knowledge to be referred to and built upon, as well as could favor mutual cross-pollination between the two fields [71]. The artefacts of HRI exhibit autonomy and are geared to establishing relationships with people [34]. Engaging with the HRI field would enrich the discussion in the DIS community about what should be considered as an artefact and what, as interaction designers, we can learn from these complex artefacts (and the novel relationships they do and could build with humans) where it is difficult to disentangle design and engineering [71]. 


\section{REFERENCES}

[1] Sigurdur Orn Adalgeirsson and Cynthia Breazeal. 2010. MeBot: A robotic platform for socially embodied telepresence. In 2010 th ACM/IEEE International Conference on Human-Robot Interaction (HRI). IEEE, 15-22. https://doi.org/10. 1109/HRI.2010.5453272

[2] Henny Admoni and Brian Scassellati. 2017. Social eye gaze in human-robot interaction: a review. Journal of Human-Robot Interaction 6, 1 (2017), 25-63. https://doi.org/10.5898/JHRI.6.1.Admoni

[3] Patrícia Alves-Oliveira, Patrícia Arriaga, Ana Paiva, and Guy Hoffman. 2017 Yolo, a robot for creativity: A co-design study with children. In Proceedings of the 2017 Conference on Interaction Design and Children. 423-429. https: //doi.org/10.1145/3078072.3084304

[4] Lucy Anderson-Bashan, Benny Megidish, Hadas Erel, Iddo Wald, Guy Hoffman, Oren Zuckerman, and Andrey Grishko. 2018. The greeting machine: an abstract robotic object for opening encounters. In 2018 27th IEEE International Symposium on Robot and Human Interactive Communication (RO-MAN). IEEE, 595-602. https://doi.org/DOI:10.1109/ROMAN.2018.8525516

[5] Sean Andrist, Michael Gleicher, and Bilge Mutlu. 2017. Looking coordinated Bidirectional gaze mechanisms for collaborative interaction with virtual charac ters. In Proceedings of the 2017 CHI conference on human factors in computing systems. 2571-2582. https://doi.org/10.1145/3025453.3026033

[6] Sean Andrist, Xiang Zhi Tan, Michael Gleicher, and Bilge Mutlu. 2014. Conversational gaze aversion for humanlike robots. In 2014 9th ACM/IEEE International Conference on Human-Robot Interaction (HRI). IEEE, 25-32.

[7] Theo Araujo. 2018. Living up to the chatbot hype: The influence of anthropomorphic design cues and communicative agency framing on conversational agent and company perceptions. Computers in Human Behavior 85 (2018), 183-189. https://doi.org/10.1016/j.chb.2018.03.051

[8] James Auger. 2014. Living with robots: A speculative design approach. fournal of Human-Robot Interaction 3, 1 (2014), 20-42.

[9] Laura Barbier and Valerie Fointiat. 2020. To Be or Not Be Human-Like in Virtual World. Frontiers in Computer Science 2 (2020), 15. https://doi.org/10.3389/fcomp. 2020.00015

[10] Jeffrey Bardzell, Shaowen Bardzell, Peter Dalsgaard, Shad Gross, and Kim Halskov. 2016. Documenting the research through design process. In Proceedings of the 2016 ACM Conference on Designing Interactive Systems. 96-107. https://doi.org/10.1145/2901790.2901859

[11] Wolmet Barendregt, Olof Torgersson, Eva Eriksson, and Peter Börjesson. 2017 Intermediate-level knowledge in child-computer interaction: A call for action. In Proceedings of the 2017 Conference on Interaction Design and Children. 7-16.

[12] Christoph Bartneck. 2017. Reviewers' scores do not predict impact: bibliometric analysis of the proceedings of the human-robot interaction conference. Scientometrics 110, 1 (2017), 179-194. https://doi.org/10.1145/2317956.2317967

[13] Christoph Bartneck, Tony Belpaeme, Friederike Eyssel, Takayuki Kanda, Merel Keijsers, and Selma Šabanović. 2020. Human-robot interaction: An introduction. Cambridge University Press.

[14] Ditte Amund Basballe and Kim Halskov. 2012. Dynamics of research through design. In Proceedings of the Designing Interactive Systems Conference. 58-67.

[15] Paul Baxter, James Kennedy, Emmanuel Senft, Severin Lemaignan, and Tony Belpaeme. 2016. From characterising three years of HRI to methodology and reporting recommendations. In 2016 11th ACM/IEEE International Conference on Human-Robot Interaction (HRI). IEEE, 391-398. https://doi.org/10.1109/HRI 2016.7451777

[16] Jordan Beck and Laureline Chiapello. 2018. Schön's intellectual legacy: A citation analysis of DRS publications (2010-2016). Design Studies 56 (2018), 205-224 https://doi.org/10.1016/j.destud.2017.10.005

[17] Michael AR Biggs and Daniela Büchler. 2007. Rigor and practice-based research. Design issues 23, 3 (2007), 62-69.

[18] Ian Bogost. 2012. Alien phenomenology, or, what it's like to be a thing. U of Minnesota Press.

[19] Cynthia Breazeal. 2000. Proto-conversations with an anthropomorphic robot In Proceedings 9th IEEE International Workshop on Robot and Human Interactive Communication. IEEE RO-MAN 2000 (Cat. No. 00TH8499). IEEE, 328-333. https: //doi.org/doi:10.1109/ROMAN.2000.892518

[20] Philip J Cash. 2018. Developing theory-driven design research. Design Studies 56 (2018), 84-119.

[21] Kah-Hin Chai and Xin Xiao. 2012. Understanding design research: A bibliometric analysis of Design Studies (1996-2010). Design Studies 33, 1 (2012), 24-43.

[22] Nazli Cila, Iskander Smit, Elisa Giaccardi, and Ben Kröse. 2017. Products as agents: Metaphors for designing the products of the IoT age. In Proceedings of the 2017 CHI Conference on Human Factors in Computing Systems. 448-459.

[23] Caroline Claisse, Daniela Petrelli, and Nick Dulake. 2019. Design synthesis: an act of Research through Design. In Proceedings of the 4th Biennial Research Through Design Conference. Newcastle University. https://doi.org/10.6084/m9. figshare.7855826.v1

[24] Herbert H Clark and Susan E Brennan. 1991. Grounding in communication. (1991). https://doi.org/10.1037/10096-006
[25] Rachel Cooper. 2019. Design research-Its 50-year transformation. Design Studies 65 (2019), 6-17.

[26] Catherine Durnell Cramton. 2002. Finding common ground in dispersed collaboration. Organizational dynamics 30, 4 (2002).

[27] Nigel Cross. 2001. Designerly ways of knowing: Design discipline versus design science. Design issues 17, 3 (2001), 49-55.

[28] Nigel Cross. 2018. Developing design as a discipline. Journal of Engineering Design 29, 12 (2018), 691-708.

[29] Alma Leora Culén, Jorun Børsting, and William Gaver. 2020. Strategies for Annotating Portfolios: Mapping Designs for New Domains. In Proceedings of the 2020 ACM Designing Interactive Systems Conference. 1633-1645. https: /doi.org/10.1145/3357236.3395490

[30] Peter Dalsgaard and Christian Dindler. 2014. Between theory and practice: bridging concepts in HCI research. In Proceedings of the SIGCHI conference on Human Factors in Computing Systems. 1635-1644. https://doi.org/10.1145/ 2556288.2557342

[31] Kerstin Dautenhahn. 2007. Methodology \& themes of human-robot interaction: A growing research field. International fournal of Advanced Robotic Systems 4, 1 (2007), 15

[32] Kerstin Dautenhahn. 2018. Some Brief Thoughts on the Past and Future of Human-Robot Interaction. F. Hum.-Robot Interact. 7, 1, Article 4 (May 2018), 3 pages. https://doi.org/10.1145/3209769

[33] Kerstin Dautenhahn. 2018. Some brief thoughts on the past and future of human-robot interaction.

[34] Kerstin Dautenhahn, Bernard Ogden, and Tom Quick. 2002. From embodied to socially embedded agents-implications for interaction-aware robots. Cognitive Systems Research 3, 3 (2002), 397-428.

[35] Audrey Desjardins and Cayla Key. 2020. Parallels, Tangents, and Loops: Reflections on the'Through'Part of RtD. In Proceedings of the 2020 ACM Designing Interactive Systems Conference. 2133-2147.

[36] Carl DiSalvo and Jonathan Lukens. 2011. Nonanthropocentrism and the nonhuman in design: possibilities for designing new forms of engagement with and through technology. From social butterfly to engaged citizen: urban informatics, social media, ubiquitous computing, and mobile technology to support citizen engagement 421 (2011)

[37] Anthony Dunne and Fiona Raby. 2007. Technological dreams series: No. 1, robots. Retrieved Sep 20 (2007), 2016.

[38] Pelle Ehn. 2008. Participation in design things. In Participatory Design Conference (PDC), Bloomington, Indiana, USA (2008). ACM Digital Library, 92-101.

[39] Boris Ewenstein and Jennifer Whyte. 2009. Knowledge practices in design: the role of visual representations asepistemic objects'. Organization studies 30, 1 (2009), 07-30.

[40] Robert Farrell and Cliff Hooker. 2014. Values and norms between design and science. Design Issues 30, 3 (2014), 29-38.

[41] Julia Fink, Séverin Lemaignan, Pierre Dillenbourg, Philippe Rétornaz, Florian Vaussard, Alain Berthoud, Francesco Mondada, Florian Wille, and Karmen Franinović. 2014. Which robot behavior can motivate children to tidy up their toys? Design and Evaluation of" Ranger". In Proceedings of the 2014 ACM/IEEE international conference on Human-robot interaction. 439-446. https://doi.org/ $10.1145 / 2559636.2559659$

[42] Laura Forlano. 2016. Decentering the human in the design of collaborative cities. Design Issues 32, 3 (2016), 42-54.

[43] Jodi Forlizzi and Carl DiSalvo. 2006. Service robots in the domestic environment: a study of the roomba vacuum in the home. In Proceedings of the 1st ACM SIGCHI/SIGART conference on Human-robot interaction. 258-265. https://doi. org/10.1145/1121241.1121286

[44] William Gaver. 2012. What should we expect from research through design?. In Proceedings of the SIGCHI conference on human factors in computing systems. 937-946. https://doi.org/10.1145/2207676.2208538

[45] Elisa Giaccardi and Johan Redström. 2020. Technology and more-than-human design. Design Issues 36, 4 (2020), 33-44.

[46] Elisa Giaccardi, Chris Speed, Nazli Cila, and Melissa L Caldwell. 2016. Things as co-ethnographers: Implications of a thing perspective for design and anthropology. Design anthropological futures 235 (2016).

[47] Paul Grice. 1989. Studies in the Way of Words. Harvard University Press.

[48] Graham Harman. 2011. The quadruple object. Zero Books (2011)

[49] Fritz Heider and Marianne Simmel. 1944. An experimental study of apparent behavior. The American journal of psychology 57, 2 (1944), 243-259.

[50] Peter Hodges, Stan Ruecker, Celso Scaletsky, Jaime Rivera, Roberto Faller, and Amanda Geppert. 2017. Four criteria for design theories. She fi: The fournal of Design, Economics, and Innovation 3, 1 (2017), 65-74.

[51] Randy Hodson. 1999. Analyzing documentary accounts. Number 128. Sage.

[52] Guy Hoffman and Wendy Ju. 2014. Designing robots with movement in mind. Fournal of Human-Robot Interaction 3, 1 (2014), 91-122.

[53] Guy Hoffman, Oren Zuckerman, Gilad Hirschberger, Michal Luria, and Tal ShaniSherman. 2015. Design and evaluation of a peripheral robotic conversation companion. In 2015 10th ACM/IEEE International Conference on Human-Robot Interaction (HRI). IEEE, 3-10. 
[54] Kristina Höök and Jonas Löwgren. 2012. Strong concepts: Intermediate-level knowledge in interaction design research. ACM Transactions on Computer Human Interaction (TOCHI) 19, 3 (2012), 1-18. https://doi.org/10.1145/2362364. 2362371

[55] Chien-Ming Huang and Bilge Mutlu. 2014. Learning-based modeling of multimodal behaviors for humanlike robots. In 20149 th ACM/IEEE International Conference on Human-Robot Interaction (HRI). IEEE, 57-64.

[56] Hiroshi Ishiguro. 2016. Android science. In Cognitive Neuroscience Robotics A Springer, 193-234.

[57] Tom Jenkins, Christopher A Le Dantec, Carl Disalvo, Thomas Lodato, and Mariam Asad. 2016. Object-oriented publics. In Proceedings of the $2016 \mathrm{CH}$ Conference on Human Factors in Computing Systems. 827-839. https://doi.org/ $10.1145 / 2858036.2858565$

[58] Malte F Jung. 2017. Affective grounding in human-robot interaction. In 2017 12th ACM/IEEE International Conference on Human-Robot Interaction (HRI. IEEE, 263-273.

[59] Peter H Kahn, Nathan G Freier, Takayuki Kanda, Hiroshi Ishiguro, Jolina H Ruckert, Rachel L Severson, and Shaun K Kane. 2008. Design patterns for sociality in human-robot interaction. In Proceedings of the 3rd ACM/IEEE international conference on Human robot interaction. 97-104. https://doi.org/10.1145/1349822. 1349836

[60] Victor Kaptelinin and Bonnie A Nardi. 2006. Acting with technology: Activity theory and interaction design. MIT press.

[61] David Kirk, Tom Rodden, and Danaë Stanton Fraser. 2007. Turn it this way: grounding collaborative action with remote gestures. In Proceedings of the SIGCHI conference on Human Factors in Computing Systems. 1039-1048.

[62] Ilpo Koskinen, John Zimmerman, Thomas Binder, Johan Redstrom, and Stephan Wensveen. 2011. Design research through practice: From the lab, field, and show room. Elsevier.

[63] Hideki Kozima, Marek P Michalowski, and Cocoro Nakagawa. 2009. Keepon. International fournal of Social Robotics 1, 1 (2009), 3-18.

[64] Bruno Latour et al. 2005. Reassembling the social: An introduction to actornetwork-theory. Oxford university press.

[65] John Law. 1992. Notes on the theory of the actor-network: Ordering, strategy, and heterogeneity. Systems practice 5, 4 (1992), 379-393.

[66] Wen-Ying Lee and Malte Jung. 2020. Ludic-HRI: Designing Playful Experiences with Robots. In Companion of the 2020 ACM/IEEE International Conference on Human-Robot Interaction. 582-584

[67] Séverin Lemaignan, Raquel Ros, E Akin Sisbot, Rachid Alami, and Michael Beetz 2012. Grounding the interaction: Anchoring situated discourse in everyday human-robot interaction. International fournal of Social Robotics 4, 2 (2012), 181-199.

[68] Peter Lloyd. 2017. From design methods to future-focused thinking: 50 years of design research. Design studies 48 (2017), A1-A8.

[69] Rachael Luck. 2019. Design research, architectural research, architectural design research: An argument on disciplinarity and identity. Design Studies 65 (2019) $152-166$.

[70] Maria Luce Lupetti. 2017. Shybo-design of a research artefact for human-robot interaction studies. Journal of Science and Technology of the Arts 9, 1 (2017), 57-69. https://doi.org/10.7559/citarj.v9i1.303

[71] Maria Luce Lupetti, Cristina Zaga, and Nazli Cila. 2021. Designerly ways of knowing in HRI: Broadening the scope of design-oriented HRI through the concept of intermediate-level knowledge. In Proceedings of the 2021 ACM/IEEE International Conference on Human-Robot Interaction. 389-398.

[72] Michal Luria, John Zimmerman, and Jodi Forlizzi. 2019. Championing Research through design in HRI. arXiv preprint arXiv:1908.07572 (2019).

[73] Ingo Lütkebohle, Frank Hegel, Simon Schulz, Matthias Hackel, Britta Wrede, Sven Wachsmuth, and Gerhard Sagerer. 2010. The Bielefeld anthropomorphic robot head "Flobi". In 2010 IEEE International Conference on Robotics and Automation. IEEE, 3384-3391.

[74] Henk Moed F. 2005. Citation analysis in research evaluation. In Current Science. Jstor, 1990-1996.

[75] Masahiro Mori, Karl F MacDorman, and Norri Kageki. 2012. The uncanny valley [from the field]. IEEE Robotics \& Automation Magazine 19, 2 (2012), 98-100.

[76] Dave Murray-Rust and Rocio von Jungenfeld. 2017. Thinking through robotic imaginaries. (2017).

[77] Bilge Mutlu, Toshiyuki Shiwa, Takayuki Kanda, Hiroshi Ishiguro, and Norihiro Hagita. 2009. Footing in human-robot conversations: how robots might shape participant roles using gaze cues. In Proceedings of the 4th ACM/IEEE international conference on Human robot interaction. 61-68.

[78] Harold G Nelson and Erik Stolterman. 2014. The design way: Intentional change in an unpredictable world. MIT press.

[79] James Pierce. 2014. On the presentation and production of design research artifacts in HCI. In Proceedings of the 2014 conference on Designing interactive systems. $735-744$.

[80] Johan Redström. 2017. Making design theory. MIT Press.

[81] David J Roedl and Erik Stolterman. 2013. Design research at CHI and its applicability to design practice. In Proceedings of the SIGCHI Conference on
Human Factors in Computing Systems. 1951-1954

[82] Raquel Ros, Séverin Lemaignan, E Akin Sisbot, Rachid Alami, Jasmin Steinwender, Katharina Hamann, and Felix Warneken. 2010. Which one? grounding the referent based on efficient human-robot interaction. In 19th International Symposium in Robot and Human Interactive Communication. IEEE, 570-575.

[83] Marco Rozendaal. 2016. Objects with intent: a new paradigm for interaction design. Interactions 23, 3 (2016), 62-65.

[84] Zoë Sadokierski. 2020. Developing critical documentation practices for design researchers. Design Studies 69 (2020), 100940.

[85] Martin Saerbeck and Christoph Bartneck. 2010. Perception of affect elicited by robot motion. In 2010 th ACM/IEEE International Conference on Human-Robot Interaction (HRI). IEEE, 53-60.

[86] Satoru Satake, Takayuki Kanda, Dylan F Glas, Michita Imai, Hiroshi Ishiguro, and Norihiro Hagita. 2009. How to approach humans? Strategies for social robots to initiate interaction. In Proceedings of the 4th ACM/IEEE international conference on Human robot interaction. 109-116.

[87] Allison Sauppé and Bilge Mutlu. 2014. Robot deictics: How gesture and context shape referential communication. In 2014 9th ACM/IEEE International Conference on Human-Robot Interaction (HRI). IEEE, 342-349.

[88] Jean C Scholtz. 2002. Human-robot interactions: Creating synergistic cyber forces. In Multi-robot systems: From swarms to intelligent automata. Springer, 177-184.

[89] Julie Shah, James Wiken, Brian Williams, and Cynthia Breazeal. 2011. Improved human-robot team performance using chaski, a human-inspired plan execution system. In Proceedings of the 6th international conference on Human-robot interaction. 29-36.

[90] David Sirkin, Brian Mok, Stephen Yang, and Wendy Ju. 2015. Mechanical ottoman: how robotic furniture offers and withdraws support. In Proceedings of the Tenth Annual ACM/IEEE International Conference on Human-Robot Interaction. $11-18$.

[91] Sichao Song and Seiji Yamada. 2017. Expressing emotions through color, sound, and vibration with an appearance-constrained social robot. In 2017 12th ACM/IEEE International Conference on Human-Robot Interaction (HRI. IEEE, 2-11.

[92] Pieter Stappers and Elisa Giaccardi. 2017. Research through design. The encyclopedia of human-computer interaction 2 (2017).

[93] Erik Stolterman and Mikael Wiberg. 2010. Concept-driven interaction design research. Human-Computer Interaction 25, 2 (2010), 95-118.

[94] Daniel Szafir, Bilge Mutlu, and Terrence Fong. 2014. Communication of intent in assistive free flyers. In Proceedings of the 2014 ACM/IEEE international conference on Human-robot interaction. 358-365.

[95] Daniel Szafir, Bilge Mutlu, and Terrence Fong. 2015. Communicating directionality in flying robots. In 2015 10th ACM/IEEE International Conference on Human-Robot Interaction (HRI). IEEE, 19-26.

[96] Hamish Tennent, Solace Shen, and Malte Jung. 2019. Micbot: A peripheral robotic object to shape conversational dynamics and team performance. In 2019 14th ACM/IEEE International Conference on Human-Robot Interaction (HRI). IEEE, 133-142.

[97] Michael Tomasello. 2010. Origins of human communication. MIT press.

[98] Gabriele Trovato, Cesar Lucho, Alexander Huerta-Mercado, and Francisco Cuellar. 2018. Design strategies for representing the divine in robots. In Companion of the 2018 ACM/IEEE International Conference on Human-Robot Interaction. 29-35.

[99] Pei-Yun Tu, Chien Wen Yuan, and Hao-Chuan Wang. 2018. Do you think what I think: Perceptions of delayed instant messages in computer-mediated communication of romantic relations. In Proceedings of the 2018 CHI Conference on Human Factors in Computing Systems. 1-11.

[100] Ron Wakkary, Doenja Oogjes, Sabrina Hauser, Henry Lin, Cheng Cao, Leo Ma, and Tijs Duel. 2017. Morse things: A design inquiry into the gap between things and us. In Proceedings of the 2017 conference on designing interactive systems. 503-514.

[101] Stephan Wensveen and Ben Matthews. 2015. Prototypes and prototyping in design research. The Routledge Companion to Design Research. Taylor \& Francis (2015).

[102] Cristina Zaga. 2021. The Design of Robothings: Non-Anthropomorphic and Non-Verbal Robots to Promote Children's Collaboration Through Play. (2021). https://doi.org/10.3990/1.9789036551380

[103] Cristina Zaga, Roelof AJ de Vries, Jamy Li, Khiet P Truong, and Vanessa Evers. 2017. A simple nod of the head: The effect of minimal robot movements on children's perception of a low-anthropomorphic robot. In Proceedings of the 2017 CHI conference on human factors in computing systems. 336-341. https: //doi.org/10.1145/3025453.3025995

[104] John Zimmerman, Jodi Forlizzi, and Shelley Evenson. 2007. Research through design as a method for interaction design research in HCI. In Proceedings of the SIGCHI conference on Human factors in computing systems. 493-502. https: //doi.org/10.1145/1240624.1240704

[105] Jakub Złotowski, Diane Proudfoot, Kumar Yogeeswaran, and Christoph Bartneck. 2015. Anthropomorphism: opportunities and challenges in human-robot interaction. International journal of social robotics 7, 3 (2015), 347-360. 